\title{
Notes: The Possibility of Mass Shift into The Past
}

\author{
Anthony Maccini \\ Flat 9, Fairhall Court, 114 - 124 Kingcharles Road, Surbiton, KT5 8QL, England
E-mail: anthonymaccini@hotmail.com
}

corresponding author details: Anthony Maccini; anthonymaccini@hotmail.com

\begin{abstract}
This paper considers the work of two scientists, Darko Bajlo who first detected advanced waves in a set of experiments on 3rd December 2016 to 5th January 2017 and Talaaki Musha experiments on 1st February until 1st March in 1996, who got weight reduction of a capacitor by feeding into it an electromagnetic field. From both their work I see the possibility of an experiment of combining both experiments to send advanced waves into a capacitor to see if the mass shift of the capacitor is shifted into the past.
\end{abstract}

Keywords: advanced waves; capacitor; electromagnetic field; mass shift; retarded waves; weight reduction

\section{INTRODUCTION}

The possibility of mass shift of an object into the past opens up the prospect of time travel. Indeed, advanced effects have been found in the work of Talaaki Musha in a number of experiments, where he shows in this paper that Photons travel at superluminal speed as an electromagnetic near field of the source [1].

E. Recami claimed that tunneling Photons can move with superluminal speed [2]. Chu and S. Wang at BT Bell labs measured superluminal velocities for light [3]. Steinberg, Kwait and Chiao did an experiment, measured the tunneling time of light with an optical filter, confirmed superluminal speed [4].

The work of Wang, Kuzmuch A, Dogariu A, who did optical experiments at Princeton NEC have verified superluminal propagation in transparent media [5]. And walker WD has shown that fields generated by an electric or magnetic dipole propagate superluminally in the nearfield of the source and reduce to the speed of light as they move into the far field. That he found information signal can be propagated superluminally, that it can be reflected by a moving frame and arrive at the source before the information was transmitted, enabling causality to be violated [6]. So, there is evidence here of advanced phenomena, but is not generally believed by the mainstream scientific community nor the public in general, mostly because this is due to a lack of awareness of such work, nevertheless such experiments have been done. What was found in most of these experiments is of the effect arising before the cause of electromagnetic waves and of light travelling faster than light. Is it any surprise then that Darko Bajlo's experiment is first in detecting advanced waves? [7]. The advanced waves arise before the detection of the retarded waves.

The prospect of feeding such advanced waves into a capacitor, that the shift of mass in its weight reduction into the past has not yet been done. Indeed, there has been much work in mass shift, weight reduction and forces detected from capacitors. T. T. Brown (Known as the Biefeld-Brown effect, B-B) found with a charged capacitor with dielectrics detected unidirectional thrust to a positive plate [8]. There is also the work of Talaaki Musha in observed weight reduction of a dielectric [9]. D. R. Bueler observed a net force upon a capacitor mass [10].

\section{ADVANCED WAVES}

Around 2019, I obtained the papers of experiments of Darko Bajlo [8][11]. On the measurement of advanced electromagnetic radiation, who appears to be the first person to have detected advanced waves. Retarded waves are normal radio waves, that travel at the speed of light. Advanced waves by contrast travel into the past. Maxwell equation's for electromagnetic waves predict two solutions, advanced waves and retarded waves.

As Bajlo says in his paper, detecting advanced electromagnetic radiation predicted by Wheeler-Feynman absorber theory for the case of incomplete absorption of retarded electromagnetic radiation, pulses in duration of $6 \mathrm{~ns}$ to $24 \mathrm{~ns}$, wavelength from $91 \mathrm{~cm}$ to $200 \mathrm{~cm}$ where supplied to three different transmitting antennas. Detection was done with a monopole antenna in the advanced time window at a time $2 \mathrm{r} / \mathrm{c}$ before the arrival of the centre of the retarded pulse. At distances ranging from $430 \mathrm{~cm}$ to $18 \mathrm{~m}$, advanced signals were measured in the SNR range from 15.4 to 30.9. From the period from 10th April 2016 to 30th August 2016 at 2000 runs no statistically significant signal above the level of the noise was detected. From the period from 3rd December 2016 to 5th January 2017 at wavelengths ranging from $91 \mathrm{~cm}$ to $200 \mathrm{~cm}$ was used. First clear signal was observed on the 2rd day after which tests were carried out with the purpose of detecting a possible source of systematic error.

As Bajlo explains the fact that he detected advanced waves, was only possible, because the receiving antenna had to be smaller than the transmitting antenna, the fact that advanced waves are not usually detected, in that the retarded signal usually cancels out the advanced waves, but by using a smaller receiving antenna the advanced waves are not cancelled out, this explains why advanced waves are not usually detected. As Bajlo says in his own words, page 5 of his paper [11]: The solution to this measurement problem 
is to minimize the influence of the measuring instrument on the phenomenon being measured. This can be easily achieved with the meter and decimeter radio waves by using a small enough receiving antenna for detection, where its advanced waves cannot completely cancel advanced waves from the transmitting antenna, if the results of the recent experiment represent the real signal and not some systematic error. Then the advanced radiation can actually be detected with a receiving antenna twenty times smaller than the wavelength of emitted electromagnetic pulse [11].

\section{WEIGHT REDUCTION}

Then I came across the work of Takkaaki Musha in JBIS 2008[9]. He says in his paper: The research group of the HONDA R\&D Institute observed a weight reduction by applying alternating electric field to a capacitor. This phenomenon, which is called the dynamical Biefeld-Brown effect, cannot be explained within the framework of conventional physics.

From the standpoint of ZPF field, the author tries to explain this phenomenon as an interaction between the vacuum electromagnetic zero-point field and the high potential electric field. Takaaki Musha further says in his paper that from the 1st February until the 2rd March 1996, the research group of HONDA R\&D institute conducted experiments to verify the B-B effect with and improved experimental device which rejected the influence of corona discharges and electric wind around the capacitor, by setting the capacitor in the insulator oil contained within a metallic vessel.

The capacitor used at the experiment was a circular plate made of high permittivity dielectric glass with the thickness $1 \mathrm{~mm}$, the diameter $170 \mathrm{~mm}$ and the weight $62 \mathrm{~g}$. The electric balance used for the experiment had the resolution of $1 \mathrm{mg}$. They conducted experiments for two cases, DC- $18 \mathrm{Kv}$ and AC-8Kv pulses supplied to the capacitor by the experimental set up. The AC voltage was supplied to the capacitor through the ignition coil to produce rectangular pulses with the repetition of $50 \mathrm{~Hz}$. After amplifying AC pulses by the ignition coil, the impulsive electric field was applied to the capacitor through the diode by changing the polarity to produce minus-biased or plus-biased voltage to the capacitor.

From the experimental results it was found that the case of AC pulses exhibited higher reduction of weight than the case of DC exposures. Maximum weight reduction measured at the experiment by applying AC pulses was $1.92 \mathrm{~g}$, which was about $3 \%$ of the own weight of the capacitor used at the experiment. So, they got positive results, they ruled out ion transfer because the estimated equations for ion transfer gave negligible small results compared with the experimental results. So, then they considered that the external electromagnetic field was responsible for the mass shift of the capacitor. Takaaki Musha concluded in his paper that the dielectric material may produce a sufficient artificial gravity to attain velocities comparable to chemical rockets. Because, this was the aim of his experiment.

\section{COMBINATION OF BOTH EXPERIMENTS}

It occurred to me, that one could combine Takaaki Musha's experiment of weight reduction with that of Bajlo's experiment of producing advanced waves, that instead of applying a high intensity field to the capacitor for weight loss of the dielectric, that one may try to achieve the same effect of weight loss by applying high intensity advanced field to the capacitor, of the dielectric to achieve weight loss by the arrangement of Bajlos experiment. What would the effect be of advanced waves or high intensity advanced field have on the capacitor. The fact that such and advanced field is travelling into the past, would it effect the dielectric for its mass shift to be shifted into the past?
The antenna in the arrangement of Bajlo's experiment has to be combined with Takaaki Musha's experimental arrangement of the condenser, when one feeds advanced intensity field into the capacitor. Of course, its as yet unknown as yet what would happen, would the mass of the dielectric be shifted into the past, the only way of knowing is until such a experiment is done. Of course, one would have to repeat the same experiment of Bajlo's to prove to yourself if indeed you detect advanced electromagnetic radiation. Bajlo used RF signal generator to generate pulses, that could take place in the same arrangement in weight reduction experiment where AC pulses were used, that had a higher reduction of weight. The conditions of receiving advanced waves in Bajlo's experiment, was that advanced waves may bedetectable if the impact of the measuring instrument on the phenomena being measured is minimized, as recent experiments with radio waves has indicated.

\section{CONCLUSION}

Here is a possibility of doing a experiment to test the possibility if an object can be shifted into the past, opens the door to the undreamt possibility of time travel. But were never know unless a experiment is actually done, for then we will know if it is possible or not. It's because advanced waves are travelling into the past, that this direction may effect the mass shift of the dielectric to shift into the direction of the past.

\section{REFERENCES}

[1] Musha T, (2019) Superluminal Speed of Photons in the Electromagnetic Near-Field. Recent Advances in Photonics and Optics, Vol 2, Issue 1, Pages 36-39.

[2] Recami E (2001) A bird's-eye view of the experimental status-of-the-art for superluminal motions. Foundation of Physics 32:1119-1135.

[3] Brown J (1995) Faster than the speed of light. New Scientist 146: 26-30.

[4] Steinberg AM, Kwait PG, Chiao RY (1993) Measurment of single-photon tunneling time. Physical Review letters 71: 708-711.

[5] Wang, Kuzmich A, Dogariu A (2000) Gain-assisted superluminal light propagation. Nature 406:277-279.

[6] Walker WD, Superluminal Electromagnetic and Gravitational Fields generated in the Nearfield of Dipole Sources. Norwegian University of Science and Technology (NTNU) P1-35.

[7] Bajlo D. (2017). Measurement of Advanced Electromagnetic radiation. Zenodo doi: 10,5281/zenodo. 247283.

[8] INTEL, (1956) 'Toward Flight without Stress or Strain...or Weight", Interavia XI, pp.373-374.

[9] Musha T, (2008) Explanation of dynamical BiefeldBrown effect from the standpoint of ZPF Field. JBIS, Vol. 61, pp.379-389, (2008).

[10] D.R. Buehler, (2004) "Exploratory Research on the Phenomenon of the Movement of High Voltage Capacitors", Journal of Space Mixing, 2, pp.1-22.

[11] Bajlo D, (September 2017) The hidden arrow of electromagnetic radiation: unmasking aadvanced waves. 